\title{
La gravedad del prurito y su relación con la edad e índice de masa corporal en pacientes con enfermedad renal crónica en terapia de reemplazo renal
}

\author{
The severity of pruritus and its relationship to age and body mass index \\ in patients with chronic kidney disease on renal replacement therapy
}

\author{
Adriana Vergara Suárez, * Rafael Valdez Ortiz, ${ }^{\ddagger}$ Nathan Berman Parks, ${ }^{\S}$ Alejandro Ezquerra Osorio, \\ Grecia Figueroa Ramos, " Débora Salero Martínez, "Alain Sánchez Rodríguez**
}

\begin{abstract}
Citar como: Vergara SA, Valdez OR, Berman PN, Ezquerra OA, Figueroa RG, Salero MD et al. La gravedad del prurito y su relación con la edad e índice de masa corporal en pacientes con enfermedad renal crónica en terapia de reemplazo renal. Acta Med Grupo Angeles. 2021; 19 (2): 208-213. https://dx.doi.org/10.35366/100444
\end{abstract}

\section{Resumen}

Introducción: Se desconocen los factores asociados a prurito urémico grave y el impacto sobre la calidad del sueño en pacientes con enfermedad renal crónica (ERC) en terapia de reemplazo renal (TRR) en México. Objetivo: Correlacionar características clínicas y bioquímicas con la gravedad del prurito urémico en pacientes con ERC en TRR. Material y métodos: Estudio descriptivo, transversal en el Hospital General de México de agosto a septiembre de 2018. Incluimos variables demográficas, bioquímicas y características del prurito urémico cuatro semanas previas a la evaluación. Se realizó estadística descriptiva y regresión logística binaria multivariada para identificar factores de riesgo para prurito grave, definido con escala visual análoga (EVA) > 6 puntos. Resultados: Se incluyeron 99 pacientes, $60.6 \%$ varones, edad promedio $44.9 \pm 4.2$. Variables asociadas a prurito grave: edad (42.4 vs $49.7, p=0.021)$ IMC (23.8 vs $\left.25.9 \mathrm{~kg} / \mathrm{m}^{2}, \mathrm{p}=0.019\right)$. Los factores asociados a prurito grave obtenidos en el análisis de regresión multivariado fueron IMC $\geq 24$ (OR = 3.37 [IC95\% 1.008-11.29, p = 0.049]), prurito diseminado (OR = 17.2 [IC95\% 1.039-286.6, $p=0.047]$ ), despertares (OR = 10.9 [IC95\% 2.21-53.87, $p=0.003])$. No se observó asociación con variables bioquímicas ni modalidad de TRR. Conclusiones: El prurito urémico grave tuvo asociación con mayor IMC, edad, presentación diseminada y despertares.

\section{Abstract}

Introduction: Information regarding severity of uremic pruritus, impact on sleep quality in patients with end stage renal disease (ESRD) in renal replacement therapy (RRT) is scarce in Mexico. Objective: To correlate clinical and biochemical features, body mass index (BMI), sleep quality impairment with severity of uremic pruritus in patients with ESRD in RRT. Material and methods: Cross sectional study with patients of "Hospital General de México" from August through September 2018. Clinical, biochemical and sleep quality variables were collected. Characteristics of pruritus four weeks before assessment was obtained. A descriptive analysis and multivariate logistic regression for severe pruritus [visual analogue scale (VAS) of $>6$ points] were analyzed. Results: Data from 99 patients was included. $60.6 \%$ male, age $44.9 \pm 4.2$ years. Patients with severe pruritus were older ( 42.4 vs 49.7 years $p=0.021$ ), had higher BMI (23.8 vs $\left.25.9 \mathrm{~kg} / \mathrm{m}^{2}, \mathrm{p}=0.019\right)$. Other associations identified were $\mathrm{BMI} \geq 24 \mathrm{~kg} / \mathrm{m}^{2}$ [OR $=3.37$ (95\% Cl 1.008$11.29, p=0.049)$ ], disseminated pruritus [OR $=17.2(95 \%$ Cl 1.039-286.6, $p=0.047)$ ]; awakenings OR $=10.9(95 \%$ Cl 2.21-53.87, $p=0.003$ ). No association was observed for biochemical variables or TRR modality. Conclusions: Severe uremic pruritus was associated with higher BMI, older age, disseminated affection and awakenings.
* Facultad Mexicana de Medicina, Universidad La Salle; Hospital Ángeles Metropolitano.

* Jefe de Servicio de Nefrología, Hospital General de México "Dr. Eduardo Liceaga".

$\S$ Nefrología y Metabolismo Mineral, Instituto Nacional de Ciencias Médicas y Nutrición "Salvador Zubirán".

" Cardiología, Instituto Nacional de Cardiología "Ignacio Chávez".

I Medicina Interna, Hospital Ángeles Metropolitano.

** Medicina Interna y Reumatología, Centro Médico ABC.
Ciudad de México.

Correspondencia:

Alain Sánchez Rodríguez

Correo electrónico: sanchezrodriguez.alain@gmail.com

Aceptado: 01-09-2020.

www.medigraphic.com/actamedica

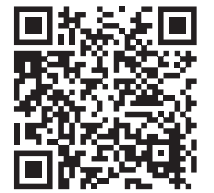


Palabras clave: Prurito urémico, enfermedad renal crónica, índice de masa corporal, calidad del sueño, calidad de vida, terapia de reemplazo renal.
Keywords: Uremic pruritus, end stage renal disease, renal replacement therapy, body mass index, sleep quality, health related quality of life, renal replacement therapy.

\section{INTRODUCCIÓN}

La enfermedad renal crónica (ERC) es una condición frecuente en México con una prevalencia aproximada de $10.2 \%$ (7.7-13.0\%), se ha asociado a incremento en la mortalidad, dificultad en el acceso a la atención médica y guarda una estrecha relación con otros factores de riesgo comunes en nuestro país como obesidad, diabetes mellitus tipo 2, hipertensión y síndrome metabólico. ${ }^{1-3}$

Un síntoma frecuente y poco reconocido en pacientes con ERC es el prurito, especialmente en etapas avanzadas de la enfermedad en sujetos en tratamiento con terapia de reemplazo renal (TRR), ya sea hemodiálisis (HD) o diálisis peritoneal (DP)., ${ }^{4,5}$ La etiología del prurito urémico es multifactorial, podría involucrar mecanismos como inflamación local, hiperparatiroidismo y alteraciones en el metabolismo mineral, elevación de sustancia P, acumulación de toxinas urémicas en piel y terminales nerviosas, xerosis y daño microvascular, lo que afecta la piel y sus anexos, y su fisiopatología es compleja. ${ }^{6,7}$

Estudios previos realizados en América y otras poblaciones han descrito una prevalencia aproximada de 63-72\% de prurito. Además, estas cohortes de sujetos en TRR por DP y HD han investigado factores de riesgo para prurito, gravedad del prurito, así como otros aspectos psicosociales, la topografía, el efecto del prurito sobre la calidad de vida y el horario de presentación. ${ }^{8-10}$ Sin embargo, los estudios en México sobre el comportamiento del prurito son escasos ${ }^{11}$ y la información sobre el efecto de variables como el índice de masa corporal (IMC), la edad, variables clínicas y bioquímicas de la ERC y su relación con la gravedad y la afección de la calidad del sueño es desconocida. Por lo que realizamos este estudio para describir el papel de estos factores y su impacto sobre la gravedad y características del prurito en pacientes con ERC en TRR del Hospital General de México.

\section{MATERIAL Y MÉTODOS}

Diseñamos un estudio descriptivo, transversal que reunió datos de pacientes ambulatorios evaluados en la consulta externa del departamento de hemodiálisis y diálisis peritoneal del servicio de nefrología del Hospital General de México durante agosto a septiembre de 2018. Se tomaron variables clínicas y demográficas, IMC por la fórmula de Quetelet, ${ }^{12}$ estudios de laboratorio y preguntas sobre la presencia y características del prurito durante las cuatro semanas previas a la evaluación. Las características evaluadas incluyeron intensidad con la escala visual análoga (EVA) del 0-10, frecuencia de alteraciones en el sueño mediante la escala de Likert y la frecuencia de despertares. Las características psicosociales evaluadas fueron el impacto sobre la convivencia y el rendimiento laboral. Se excluyeron a pacientes con requerimientos de diálisis de urgencia. Todos los pacientes firmaron un consentimiento informado y el estudio fue aprobado por el comité de bioética de la institución.

Análisis estadístico: utilizamos estadística descriptiva con medidas de tendencia central y de dispersión; las variables categóricas fueron expresadas como medidas de frecuencia absoluta y relativa y las variables lineales como media y desviación estándar (DE); para las variables con distribución normal o medianas con rangos intercuartílicos (RIQ) de acuerdo al tipo de distribución de frecuencias. Las variables numéricas fueron comparadas con la prueba t de Student para muestras independientes o la de $U$ de Mann-Whitney de acuerdo con las características de la distribución. Las variables fueron comparadas con $\chi^{2}$ o prueba exacta de Fisher. La medida de fuerza de asociación para estudios trasversales con Odds Ratio (OR) con intervalos de confianza del 95\%. El análisis multivariado incluyó la construcción de modelos de regresión logística binaria en el que la variable dependiente fue la presencia de prurito con un puntaje de EVA $>6$ puntos. Un error alfa ajustado menor de $5 \%$ a dos colas fue considerado significativo. El paquete estadístico fue STATA SE v 11.0.

\section{RESULTADOS}

Se incluyeron datos de 99 pacientes, $60.6 \%$ fue del sexo masculino y $39.4 \%$ del femenino. La edad promedio fue de $44.9 \pm 4.2$ años. El IMC fue de $24 \pm 4.2 \mathrm{~kg} / \mathrm{m}^{2}$. Sesenta y cinco punto siete por ciento se encontraba en hemodiálisis y $34.3 \%$ en diálisis peritoneal. La mediana del tiempo de evolución de la ERC fue de 13 meses (RIQ 6-36) y para el tiempo en terapia de sustitución fue de 5 meses (RIQ 1-15). Del total de los pacientes incluidos encontramos una prevalencia de $81 \%$ de aquellos que clínicamente tuvieron como síntomas rascado en $75.8 \%$ y xerosis en $77.9 \%$. La intensidad por EVA del prurito tuvo un promedio de 4.5 \pm 2.9 puntos. Recibieron tratamiento específico para el prurito con emolientes $49.5 \%$, con antihistamínicos $2 \%$ 
y con gabapentina 1\%. Las características demográficas y clínicas completas se muestran en la Tabla 1.

Identificamos un patrón de mayor intensidad sin alcanzar diferencias significativas en la EVA de intensidad del prurito medido de forma lineal a medida que aumentan la edad ( $p=0.14)$ y el IMC ( $p=0.34)$, con una prevalencia

\begin{tabular}{|c|c|}
\hline & $\mathrm{n}(\%)$ \\
\hline Edad (años) & $44.9 \pm 4.2$ \\
\hline \multicolumn{2}{|l|}{ Sexo } \\
\hline Masculino & $60(60.6)$ \\
\hline Femenino & $39(39.4)$ \\
\hline Índice de masa corporal, kg/m² & $24(4.2)$ \\
\hline \multicolumn{2}{|c|}{ Etiología de la enfermedad renal crónica } \\
\hline Diabetes & $48(48.5)$ \\
\hline HAS & $16(16.2)$ \\
\hline ERPA & $2(2.0)$ \\
\hline GMN & $2(2.0)$ \\
\hline Vasculitis & $1(1.0)$ \\
\hline Desconocida & $26(26.3)$ \\
\hline Otras & $4(4.0)$ \\
\hline \multicolumn{2}{|c|}{ Frecuencia de prurito a la semana (días) } \\
\hline Sin prurito & $17(17.2)$ \\
\hline $1-2$ & $23(23.2)$ \\
\hline $3-4$ & $24(24.2)$ \\
\hline $5-6$ & $3(3.0)$ \\
\hline 7 & $31(31.3)$ \\
\hline \multicolumn{2}{|l|}{ Topografía } \\
\hline No especificado & $6(6.1)$ \\
\hline Generalizado & $5(5.1)$ \\
\hline Diseminado & $55(55.6)$ \\
\hline Localizado & $21(21.2)$ \\
\hline \multicolumn{2}{|c|}{ Características de las excoriaciones } \\
\hline Sin excoriaciones & $2(2.0)$ \\
\hline Excoriaciones superficiales & $53(53.5)$ \\
\hline Excoriaciones profundas & $32(32.3)$ \\
\hline \multicolumn{2}{|l|}{ Frecuencia de afección del sueño } \\
\hline Nunca & $65(65.7)$ \\
\hline Rara vez & $4(4.0)$ \\
\hline A veces & $14(14.1)$ \\
\hline Casi siempre & $4(4.0)$ \\
\hline Siempre & $1(1.0)$ \\
\hline Rara vez & $1(1.1)$ \\
\hline A veces & $2(2.3)$ \\
\hline \multicolumn{2}{|l|}{ Frecuencia de afección laboral } \\
\hline Nunca & $84(84.8)$ \\
\hline Rara vez & $3(3.0)$ \\
\hline Casi siempre & $1(1.0)$ \\
\hline
\end{tabular}

HAS = hipertensión, ERPA = enfermedad renal poliquística autosómica, $\mathrm{GMN}=$ glomerulonefritis.
Figura 1: Comparación entre la proporción de pacientes con prurito entre los diferentes grupos etarios. Al comparar los porcentajes entre los diferentes grupos no se encontraron diferencias significativas $(p=0.83)$.

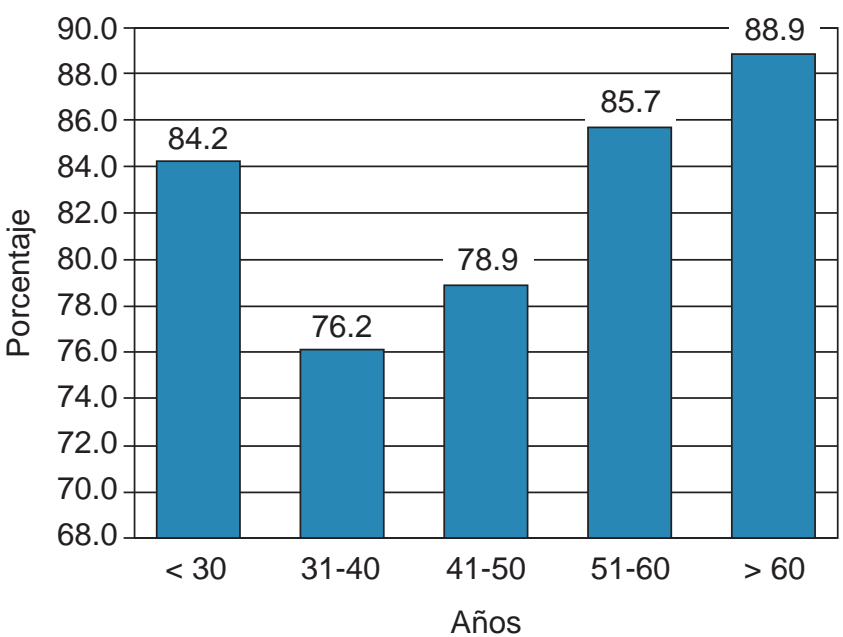

Figura 2: Comparación entre la proporción de pacientes con prurito entre los grupos estratificados por índice de masa corporal. Al comparar los porcentajes entre los diferentes grupos no se encontraron diferencias significativas $(p=0.34)$.

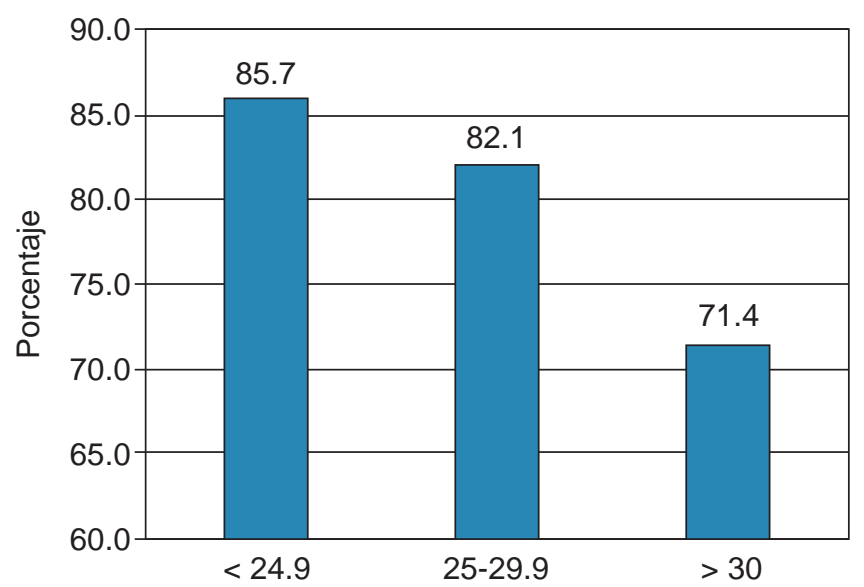

que oscila entre 76.2 a $88.9 \%$, y sin diferencias al comparar entre los grupos etarios ( $p=0.83$ ) o grupos del IMC ( $p=$ 0.44). Estas comparaciones se detallan en las Figuras 1 a 3. Las variables asociadas a prurito grave fueron la edad (42.4 vs $49.7, p=0.021)$, el peso (61.2 vs 66.7, $p=0.05$ ) y el IMC (23.8 vs 25.9, $\mathrm{p}=0.019$ ) (Figura 3). Por otro lado, no se observó correlación entre las variables que miden intensidad y frecuencia de prurito y los estudios de laboratorio. La comparación de las variables relevantes, incluidos los resultados de laboratorio, se detallan en la Tabla 2. El análisis multivariado reveló que las variables asociadas a 
prurito grave fueron la presencia de prurito diseminado OR $=17.2(\mathrm{IC} 95 \% 1.039-286.6, \mathrm{p}=0.047), \mathrm{IMC} \geq 24 \mathrm{OR}=$ $3.37($ IC95\% 1.008-11.29, $\mathrm{p}=0.049)$, despertares OR $=$ 10.9 (IC95\% 2.21-53.87, $\mathrm{p}=0.003$ ) (Tabla 3).

\section{DISCUSIÓN}

Presentamos un estudio epidemiológico que explora las características, frecuencia e intensidad del prurito urémico y sus posibles asociaciones con variables clínicas y bioquímicas en pacientes en TRR. Nuestro trabajo explora variables no previamente abordadas en estudios realizados en México. Un trabajo similar realizado anteriormente en el Hospital General de México logró demostrar que el prurito está asociado al deterioro de la calidad de vida medida por DLQI con un coeficiente de correlación de Pearson $(r)=0.47, \mathrm{Cl} 0.36-0.57,(p<0.0001) .{ }^{11}$ Por otro lado, otro estudio realizado en Perú no describió la presencia o la gravedad del prurito definida como una EVA $>7$ con el deterioro de la calidad de vida, pero sí con la presencia de hiperfosfatemia con una ratio de prevalencia (PR) de 1.71, (IC95\% 1.09-267) y el uso de antihistamínicos PR = 2.48 (IC95\% 1.56-3.94) sin existir un papel asociado con el tiempo de tratamiento con la TTR. ${ }^{8}$

Los hallazgos de nuestra investigación revelaron que los pacientes con prurito grave requieren tratamiento con mayor frecuencia y a pesar de no incluir la medición de la calidad de vida con un instrumento validado para dicho propósito, nuestros datos revelan que el prurito impacta sobre los factores psicosociales de los pacientes;

Figura 3: Comparación del índice de masa corporal entre los grupos divididos de acuerdo con la intensidad del prurito medido por escala visual análoga en $>6$ vs $<6$, con un resultado de 23.8 vs 25.9, $(p=0.019)$.

EVA = escala visual análoga.

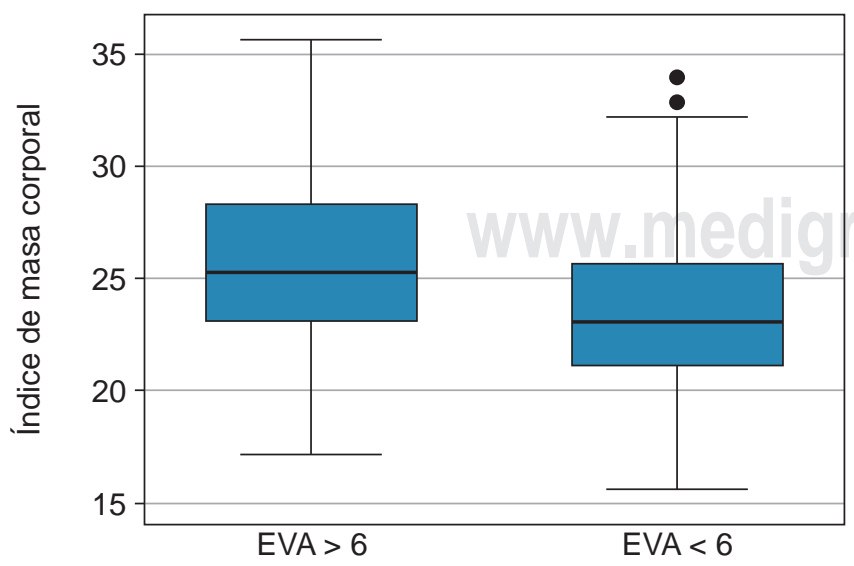

sin embargo, no logramos demostrar una diferencia con respecto a los parámetros bioquímicos relacionados con la eficiencia de la diálisis. Las teorías que explican el origen del prurito en pacientes con TRR son diversas. De forma clásica derivada de estudios realizados hace más de cuatro décadas, la teoría con mayor aceptación que explica el origen del prurito propone un mecanismo de lesión directa en los tejidos inducida por anemia, hiperparatiroidismo, acumulación de urea, hiperfosfatemia y neurotransmisores a nivel local como la sustancia $\mathrm{P}_{,}^{7,13}$ dicha teoría se ha reafirmado en protocolos de diálisis de alta eficiencia con mejoría de prurito con una Kt/V $\geq$ 1.5 que redujo la intensidad del prurito en pacientes en TRR con HD. ${ }^{14}$ No obstante, la evidencia con respecto a la modalidad y la eficiencia de la misma es contradictoria y podría explicarse por fenómenos adicionales a la TRR. $8,15,16$ Dado lo anterior, hoy en día la evidencia sugiere que existen mecanismos fisiopatológicos adicionales, como en un estudio realizado por Kimmel y colaboradores, en el que se dieron a la tarea de investigar la diferenciación de linfocitos Th1 y las citocinas intracelulares y plasmáticas en pacientes en TRR vs controles sin prurito, dicha investigación reveló que los pacientes con prurito urémico eran similares en características clínicas generales, pero presentaron un fenotipo de citocinas diferente a los controles, caracterizado por una mayor proporción de linfocitos Th1 y también marcadores de inflamación y citocinas relacionadas como proteína $C$ reactiva, interleucina 6, así como receptores CXCR3 en este grupo de pacientes, concluyendo que este fenómeno no ocurre únicamente como un fenómeno bioquímico de lesión directa como se pensaba antes, sino más bien podría ser una manifestación clínica que ocurre en el contexto de inflamación sistémica crónica. ${ }^{6}$ Otro trabajo propuso la participación de la interleucina 31 y la proteína $C$ reactiva en pacientes en TRR con HD. ${ }^{17}$

Por último, dado el contexto específico en el que realizamos nuestra investigación, es posible que otros mecanismos no tradicionales pudieran estar involucrados, es bien sabido que en México la obesidad es un factor con alta prevalencia y en pacientes con ERC se ha documentado la participación de la obesidad como un factor importante en el desarrollo de inflamación sistémica en estos pacientes; además, existe una línea de investigación nueva que explora la posible participación del adipocito como un protagonista dentro de la compleja interacción entre inflamación sistémica y ERC, así nuevas estrategias encaminadas a la investigación de la activación de la AMP quinasa, la función oxidativa del NADPH y el factor de crecimiento transformante $\beta$ podrían significar que el riñón y el adipocito comparten dicha cascada inflamatoria y estrés oxidativo y finalmente podrían explicar, al menos en parte, la fibrosis renal en 
ERC. ${ }^{18,19}$ Nuestros resultados podrían sustentarse entonces en la premisa de un mecanismo complejo y multifactorial que va mucho más allá de los resultados de laboratorio que son una variable subrogada de la eficiencia de la diálisis, el estado de la PTH revela la posible participación de un factor adicional recientemente descrito atribuible a la obesidad y otro factor suficiente, aunque no necesario, relacionado con la edad. Nuestra población tiene además algunas particularidades ligadas a la idiosincrasia de los pacientes atendidos en nuestro centro, donde habitualmente por tratarse de una institución pública de concentración que reúne a pacientes de todo el país se compone de pacientes sin acceso a seguridad social y en una situación socioeconómica vulnerable, lo cual se ha explorado en estudios previos en México. ${ }^{2}$ Dicho factor socioeconómico no está descrito como una variable de riesgo para prurito; sin embargo, la marginación social podría asociarse lógicamente con una menor búsqueda de atención médica para un síntoma que desde el punto de vista de un paciente de escasos recursos podría parecer irrelevante. No obstante, a pesar de ser un síntoma poco reconocido, ignorado o incluso desconocido desde el punto de vista epidemiológi$\mathrm{Co}^{5}{ }^{5}$ se ha demostrado que éste es grave y se correlaciona con variables como la depresión, la calidad del sueño y la calidad de vida medida por instrumentos como EQ5-D, PHQ-9 y DLQI, los cuales en conjunto sí se han asociado a mal pronóstico en cohortes prospectivas de pacientes con ERC, ${ }^{20,21}$ por lo que su diagnóstico y tratamiento podría tener un impacto sobre la mortalidad y la calidad de vida de los pacientes con ERC. ${ }^{9,22}$

Dado lo anterior, podríamos proponer que las fortalezas de nuestro trabajo son la identificación de factores de riesgo para la gravedad del prurito urémico, así como aumentar la información disponible en México; no obstante, tenemos limitaciones relacionadas con el diseño, al ser transversal y no abordar el efecto del tratamiento sobre las variables analizadas de la calidad de sueño y los factores psicosociales. Estudios ulteriores longitudinales, o incluso ensayos clínicos, podrían responder las interrogantes que sobrepasan los objetivos de nuestro trabajo.

\section{CONCLUSIONES}

El prurito urémico es una entidad muy frecuente y poco reconocida en pacientes con ERC en TTR. Su fisiopatología es compleja y podría involucrar mecanismos recientemente

Tabla 2: Comparación entre sujetos con prurito estratificado por intensidad.

\begin{tabular}{|c|c|c|c|}
\hline & $\begin{array}{c}E V A \leq 6 \\
(n=65) \\
n(\%)\end{array}$ & $\begin{array}{c}\text { EVA }>6 \\
(n=34) \\
n(\%)\end{array}$ & $p$ \\
\hline \multicolumn{4}{|l|}{ Características generales } \\
\hline Edad & $42.4 \pm 15.1$ & $49.7 \pm 13.8$ & 0.021 \\
\hline Femenino & $23(35.4)$ & $16(47.1)$ & 0.250 \\
\hline Índice de masa corporal & $23.8(4.1)$ & $25.9(4.2)$ & 0.019 \\
\hline Tiempo en ERC & $24.2(36.3)$ & $39.6(49.7)$ & 0.083 \\
\hline Hemodiálisis & $45(69.2)$ & $20(58.8)$ & 0.300 \\
\hline Tiempo en TTR & $13.5(24.8)$ & $14.1(18.6)$ & 0.900 \\
\hline \multicolumn{4}{|l|}{ Tratamiento de prurito } \\
\hline Antihistamínicos & $0(0.0)$ & $2(5.9)$ & 0.048 \\
\hline Emolientes & $28(43.1)$ & $21(61.8)$ & 0.077 \\
\hline Gabapentina & $0(0.0)$ & $1(2.9)$ & 0.160 \\
\hline \multicolumn{4}{|l|}{ Estudios de laboratorio } \\
\hline Urea, mg/dL & $170.9 \pm 87.1$ & $173.1 \pm 71.1$ & 0.450 \\
\hline Creatinina, mg/dL & $11.7 \pm 5.8$ & $11.5 \pm 5.0$ & 0.900 \\
\hline Sodio, $\mathrm{mEq} / \mathrm{L}$ & $133.6 \pm 15.6$ & $135.6 \pm 2.9$ & 0.910 \\
\hline Potasio, mEq/L & $5.1 \pm 1.4$ & $4.8 \pm 1.1$ & 0.470 \\
\hline Cloro, mEq/L & $101.1 \pm 5.2$ & $100.3 \pm 5.8$ & 0.350 \\
\hline Fósforo, mg/dL & $5.9 \pm 2.0$ & $6.3 \pm 2.8$ & 0.480 \\
\hline Calcio, $\mathrm{mg} / \mathrm{dL}$ & $7.9 \pm 1.0$ & $8.0 \pm 1.5$ & 0.450 \\
\hline Parathormona, ng/dL & $844.4 \pm 781.8$ & $877.8 \pm 439.1$ & 0.800 \\
\hline
\end{tabular}

ERC $=$ enfermedad renal crónica, TTR = terapia de reemplazo renal. 
Tabla 3: Factores asociados a la presencia de prurito grave*.

\begin{tabular}{lcl} 
& OR (IC 95\%) & $p$ \\
\hline Prurito diseminado & $17.26(1.039-286.6)$ & 0.047 \\
Edad $\geq 46$ años & $1.54(0.399-5.9)$ & 0.53 \\
Índice de masa corporal & $3.37(1.008-11.29)$ & 0.049 \\
$\geq 24 \mathrm{~kg} / \mathrm{m}^{2}$ & & \\
Excoriaciones & $2.83(0.823-9.74)$ & 0.09 \\
Xerosis & $0.67(0.120-3.78)$ & 0.65 \\
Despertares & $10.92(2.21-53.87)$ & 0.003 \\
Sexo masculino & $0.44(0.131-1.48)$ & 0.18 \\
Urea $\geq 150 \mathrm{mg} / \mathrm{dL}$ & $2.89(0.772-10.82)$ & 0.11 \\
Fósforo $\geq 7 \mathrm{mg} / \mathrm{dL}$ & $2.16(0.560-8.37)$ & 0.26 \\
& & \\
\hline
\end{tabular}

*Análisis realizado por regresión logística binaria multivariada con variable dependiente de prurito intensidad con EVA $>6$.

descritos como inflamación sistémica y obesidad. Adicionalmente, el tratamiento del prurito podría mejorar la calidad de vida, la calidad del sueño y mejorar el pronóstico de estos pacientes, por lo que el tratamiento del prurito urémico es un área de investigación en continuo desarrollo.

\section{REFERENCIAS}

1. GBD Chronic Kidney Disease Collaboration. Global, regional, and national burden of chronic kidney disease, 1990-2017: a systematic analysis for the Global Burden of Disease Study 2017. Lancet. 2020; 395 (10225): 709-733.

2. Agudelo-Botero M, González-Robledo MC, Reyes-Morales $\mathrm{H}$, Giraldo-Rodríguez L, Rojas-Russell M, Mino-León D et al. Health care trajectories and barriers to treatment for patients with end-stage renal disease without health insurance in Mexico: a mixed methods approach. Int J Equity Health. 2020; 19 (1): 90

3. Alegre-Díaz J, Herrington W, López-Cervantes M, Gnatiuc L, Ramirez $\mathrm{R}$, Hill M et al. Diabetes and cause-specific mortality in Mexico City. N Engl J Med. 2016; 375 (20): 1961-1971.

4. Torazza MC, Salomone M, Albanesi T, Deabate MC, Zina A, Triolo G. Uremic pruritus. Minerva Urol Nefrol. 1997; 49 (3): 125-132.

5. Valdez-Ortiz R, Vergara-Suárez A. Perspectiva dermatológica del prurito urémico: Un padecimiento común pero poco reconocido. Dermatol Rev Mex. 2017; 61 (6): 537-540.

6. Kimmel M, Alscher DM, Dunst R, Braun N, Machleidt C, Kiefer T et al. The role of micro-inflammation in the pathogenesis of uraemic pruritus in haemodialysis patients. Nephrol Dial Transplant. 2006; 21 (3): 749-755.
7. Cho YL, Liu HN, Huang TP, Tarng DC. Uremic pruritus: roles of parathyroid hormone and substance P. J Am Acad Dermatol. 1997; 36 (4): 538-543.

8. Kossuth-Cabrejos S, Gavino-Gutiérrez AM, Silva-Caso W. Factors associated with the severity of pruritus in patients with terminal chronic kidney disease undergoing hemodialysis in Lima, Peru. Dermatol Reports. 2020; 12 (1): 8310.

9. Shirazian S, Aina O, Park Y, Chowdhury N, Leger K, Hou L et al. Chronic kidney disease-associated pruritus: Impact on quality of life and current management challenges. Int J Nephrol Renovasc Dis. 2017; 10: 11-26.

10. Rhee EP, Guallar E, Hwang S, Kim N, Tonelli M, Moe SM et al. Prevalence and persistence of uremic symptoms in incident dialysis patients. Kidney 360. 2020; 1 (2): 86-92.

11. Maya GN, Valdez OR, Mayoral RH. Chronic kidney disease associated pruritus impact on dermatological quality of life of patients with chronic renal replacement therapy in hemodialysis from the General Hospital of Mexico. J Am Acad Dermatol. 2016; 74 (5): AB168.

12. Kataoka K. Indices of obesity derived from body weight and height. Nihon Rinsho. 1995; 53 (Suppl. 3): 147-153.

13. Hampers CL, Katz AI, Wilson RE, Merrill JP. Disappearance of "uremic" itching after subtotal parathyroidectomy. N Engl J Med. 1968; 279 (13): 695-697.

14. Ko MJ, Wu HY, Chen HY, Chiu YL, Hsu SP, Pai MF et al. Uremic pruritus, dialysis adequacy, and metabolic profiles in hemodialysis patients: a prospective 5-year cohort study. PLoS One. 2013; 8 (8): e71404.

15. Wu HY, Peng Y Sen, Chen HY, Tsai WC, Yang JY, Hsu SP et al. A comparison of uremic pruritus in patients receiving peritoneal dialysis and hemodialysis. Medicine (Baltimore). 2016; 95 (9): e2935.

16. Min J-W, Kim S-H, Kim YO, Jin DC, Song HC, Choi EJ et al. Comparison of uremic pruritus between patients undergoing hemodialysis and peritoneal dialysis. Kidney Res Clin Pract. 2016; 35 (2): 107-113.

17. Ko MJ, Peng Y Sen, Chen HY, Hsu SP, Pai MF, Yang JY et al. Interleukin-31 is associated with uremic pruritus in patients receiving hemodialysis. J Am Acad Dermatol. 2014; 71 (6): 1151-1159.e1.

18. Decleves AE, Sharma K. Obesity and kidney disease: differential effects of obesity on adipose tissue and kidney inflammation and fibrosis. Curr Opin Nephrol Hypertens. 2015; 24 (1): 28-36.

19. Markova I, Miklankova D, Hüttl M, Kacer P, Skibova J, Kucera J et al. The effect of lipotoxicity on renal dysfunction in a nonobese rat model of metabolic syndrome: a urinary proteomic approach. J Diabetes Res. 2019; 2019.

20. Satti MZ, Arshad D, Javed H, Shahroz A, Tahir Z, Ahmed MMH et al. Uremic pruritus: prevalence and impact on quality of life and depressive symptoms in hemodialysis patients. Cureus. 2019; 11 (7): e5178.

21. Pisoni RL, Wikstrom B, Elder SJ, Akizawa T, Asano Y, Keen ML et al. Pruritus in haemodialysis patients: international results from the dialysis outcomes and practice patterns study (DOPPS). Nephrol Dial Transplant. 2006; 21 (12): 3495-3505.

22. Mathur VS, Lindberg J, Germain M, Block G, Tumlin J, Smith M et al. A longitudinal study of uremic pruritus in hemodialysis patients. Clin J Am Soc Nephrol. 2010; 5 (8): 1410-1419. 